\title{
Methanol Vapor Sensing by Porous Silicon
}

\author{
O. YILmAZ ${ }^{a, b}$, E. KAYAHAN ${ }^{a, b, c}$, F. GÖREN ${ }^{a, b}$ AND F. DUMLUdAĞ ${ }^{d}$ \\ ${ }^{a}$ Kocaeli University, Electro-optic and Sys. Eng. Umuttepe, 41380, Kocaeli, Turkey \\ ${ }^{b}$ Kocaeli University, LATARUM Laboratory, 41275, Yenikoy-Kocaeli, Turkey \\ ${ }^{c}$ Kocaeli University, Hereke MYO, 41800, Hereke-Kocaeli, Turkey \\ ${ }^{d}$ Marmara University, Department of Physics, Göztepe Campus, 34722, Kadıköy, Istanbul, Turkey
}

\begin{abstract}
In this study, methanol vapor sensing of porous silicon was investigated. The porous silicon samples were anodized in HF based solution and rinsed with ethanol or deionized water after the anodisation. Porous silicon surface is very sensitive for methanol vapor sensing and the sensitivity affect from the rinsing procedure of porous silicon. The experimental results show that porous silicon is a promising candidate material for sensing methanol vapor.
\end{abstract}

DOI: $10.12693 /$ APhysPolA.125.288

PACS: 07.07.Df

\section{Introduction}

Semiconductor gas sensors are most attractive because they are compact, sensitive, low cost, and have low power consumption [1-4]. The simplest and most commonly accepted theory of the semiconductor sensor operation mechanisms is that atoms and molecules of the gases interact with semiconductor surfaces to influence surface conductivity and surface potential [5]. Surface conductivity changes are mainly due to changes in the free electron concentration due to charge exchange between adsorbed species from the gas and the semiconductor surface. The charge exchange occurs in a thin layer below the gas-solid interface. Therefore, for a high sensitivity gas detection the semiconductor must have a large specific area (surface area to volume ratio) to produce a higher charge exchange rate. This can be realized in practice by using porous materials due to their large specific area [6, 7]. Since, porous silicon (PS) has very high surface to volume ratio, it is a promising candidate for gas sensing. As a matter of fact, many authors have reported the modification of photoluminescence (PL) and electrical transport properties during exposure to volatile compounds [8]. Traditional method of detecting of the organic gas concentrations is mostly gas chromatography [9], but it is expensive and has long time. Therefore, we need new modern sensors cheap and capable quickly sensing of organic vapors and harmful gases.

Methanol vapor is very harmful to human health and it must be detected continuously in our living environment. Therefore, in this paper, it was measured the current response of PS in sensing methanol vapors. The structure of PS layer was characterized by scanning electron microscopy (SEM).

\section{Experimental}

The PS samples were prepared from $p$-type boron-doped crystal silicon wafer with resistivity of 2.5$3.5 \Omega \mathrm{cm}$. Before electrochemical etching, 1:24 volume ratio of $50 \% \mathrm{HF}$ acid and deionized (DI) water were used for cleaning and removing native oxidations of the silicon surface for $5 \mathrm{~min}$. We used an aqueous HF-ethanol solution with a $25 \%$ concentration (1:3 volume ratio of $50 \%$

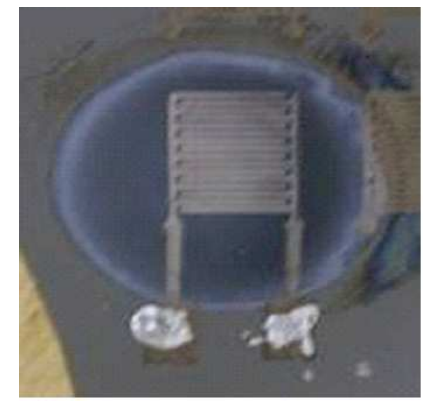

Fig. 1. Photograph of IDE coated porous silicon.

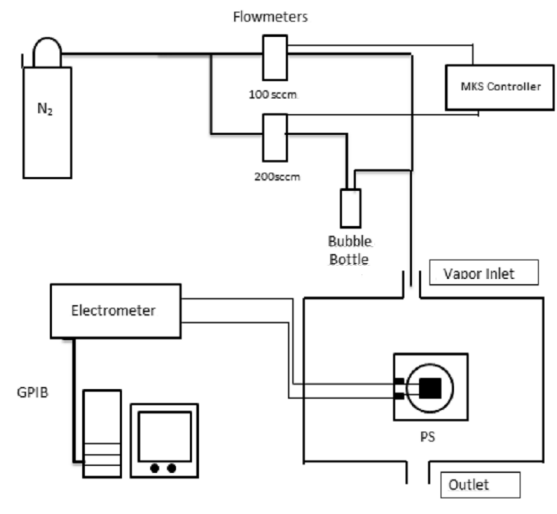

Fig. 2. Schematic shown of vapor test chamber for ethanol sensing.

hydrofluoric acid and $99.9 \%$ absolute ethanol). The contact area of the HF-ethanol solution with the silicon substrate was $1.13 \mathrm{~cm}^{2}$ and current density $8.85 \mathrm{~mA} / \mathrm{cm}^{3}$. After electrochemical etching, the PS samples were rinsed with ethanol or DI water and dried by nitrogen gas. Two PS samples were produced in the same experimental conditions; however, they were rinsed different liquids with DI water (sample 1) and pure ethanol (sample 2) for about $10 \mathrm{~s}$. After the production PS surface was coated with metal inter-digital electrode (IDE) (aluminum, $0.2 \mathrm{~g}, 99.999 \%$ ) used with thermal evaporation system. The IDE coated PS was shown in Fig. 1. Then, 
the sensor was placed in vapor test chamber for measuring electrical response of methanol vapor. The test chamber was shown in detail in Fig. 2.

During the sensor measurements, the PS samples were kept in a sealed chemical hood. The methanol vapors were obtained by flowing nitrogen gas through a bubbler and other line of nitrogen gas was used as vapor carrier. All sensor measurements were taken at room temperature.

\section{Results}

Surface morphology of the porous silicon was studied by scanning electron microscope as shown in Fig. 3. From the figure it was clear that there is a continuous distribution of pore sizes ranging between 1 and $2 \mu \mathrm{m}$.

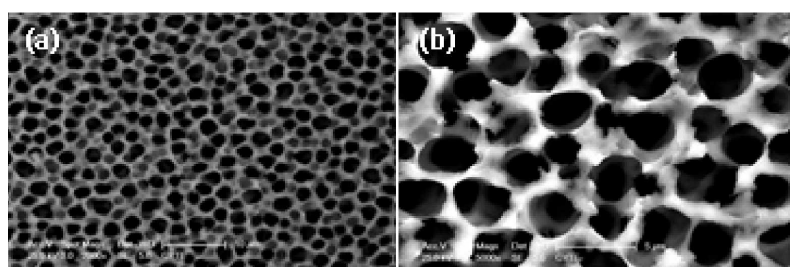

Fig. 3. SEM micrographs of the PS surface with different magnifications.



Fig. 4. Methanol vapor sensing of porous silicon produced with different rinsing methods after the anodisation, with DI water (a) and pure ethanol (b).

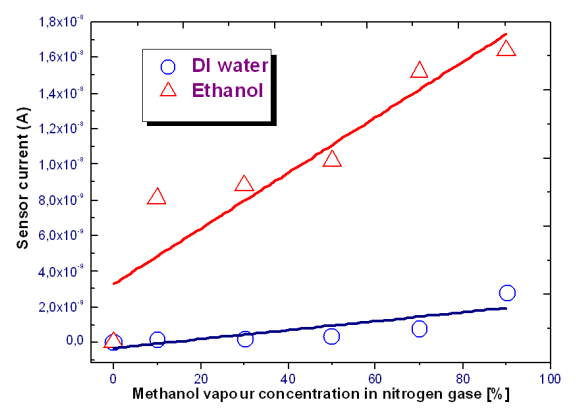

Fig. 5. The effect of ethanol and DI water rinsing of porous silicon on methanol vapor sensing. Solid line in the figure shows linear fitting.

Figure 4 shows electrical response of PS sensors during exposure to various concentrate methanol vapors. It is shown from the figure that the electrical signal increases during exposure to methanol vapor and then decreases during exposure to nitrogen gas (carrier gas). This phenomenon can be described with capillary condensation of the methanol vapors in pores of the PS [10]. During exposure to methanol vapor, air in the pores of the PS is replaced with methanol vapor. Therefore, we can see electrical signal increase due to the phenomenon of the capillary condensation.

Figure 5 shows the variation of PS methanol sensing at different methanol concentrations with ethanol or DI water rinsing of PS. As seen in the figure, it is clear that ethanol rinsing increases methanol sensitivity of PS surface. The increasing sensitivity can be explained with surface bond configurations of PS. It is well known that PS surface has mostly $\mathrm{Si}-\mathrm{H}$ bonds after the ethanol rinsing as compared with DI water rinsing. Therefore, the rinsing method affects surface bond configuration of PS.

The quantity of methanol molecule being absorbed to PS surface depends on the amount of surface passivation with H-termination and polarity of the methanol molecule [10]. Methanol belongs to an alcoholic group and polar in nature. Therefore, $\mathrm{Si}-\mathrm{H}$ bonds plays critical role for the methanol molecule adsorption on PS surface.

\section{Conclusions}

Two samples were prepared in the same experimental condition by electrochemical anodisation technique for different rinsing method. Surface morphologies of the samples were characterized by SEM and pore size was found to be 1 and $2 \mu \mathrm{m}$. The sensitivity and selectivity of PS based sensors critically depend on PS pore morphology and surface bond configuration. Hence, rinsing method of PS samples just after the anodisation is very important.

\section{Acknowledgments}

This research is financially supported by the Scientific and Technological Research Council of Turkey (project no. 111T357).

\section{References}

[1] A. Salehi, M. Gholizade, Sensor Actuat. B 89, 173 (2003).

[2] P. Menini, F. Parret, M. Guerrero, K. Soultanica, L. Erades, A. Maisonnant, B. Chaudret, Sensor Actuact. B 103, 111 (2004).

[3] G. Salgado, T.D. Becerril, H.J. Santiesteban, E.R. Andres, Opt. Mater. 29, 51 (2006).

[4] L. Canham, Properties of Porous Silicon, INSPEC, The Institution of Electrical Engineers, London 1997.

[5] V. Polishchuk, E. Souteyrand, J.R. Marin, V.I. Strikha, V.A. Skryshevsky, Anal. Chim. Acta 375, 205 (1998).

[6] L. Seals, J.L. Gole, L.A. Tse, P.J. Hesketh, J. Appl. Phys. 91, 2519 (2002).

[7] S.E. Lewis, J.R. DeBoer, J.L. Gole, Sensor Actuat. B 122, 20 (2007).

[8] I. Schechter, M. Ben Chorin, A. Kux, Anal. Chem. 67, 3727 (1995).

[9] H.J. Kim, Y.Y. Kim, K.W. Lee, Sensor Actuat. A 165, 276 (2011).

[10] S. Dhanekar, S.S. Islam, T. Islam, A.K. Shukla, Harsh, Physica E 42, 1648 (2010). 